East African Medical Journal Vol. 86 No. 12 December 2009

TREATMENT INTERRUPTION AMONG HEAD AND NECK CANCER PATIENTS UNDERGOING RADICAL RADIOTHERAPY

M. M.Solomon, BDS, MSc, Consultant, Department of Oral and Maxillofacial Surgery, Coast Provincial General Hospital, P.O. Box 90231-80100, Mombasa, Kenya, J. F. Onyango, BDS, MSc, FDSRCS, Senior Lecturer, Department of Oral and MaxillofacialSurgery, L. O. Nyabola, MSc, Senior Lecturer, Department of Community Health, A. Opiyo, MBChB, MMed, Consultant Radioncologist, Kenyatta National Hospital, P.O. Box 20723 - 00202, Nairobi, Kenya and M. L. Chindia, BDS, MSc, FFDRCSI, Associate Professor, Department of Oral and Maxillofacial Surgery, School of Dental Sciences, University of Nairobi, P.O. Box 19676 - 00202, Nairobi, Kenya

Request for reprints to: Dr. J. F. Onyango, Department of Oral and Maxillofacial Surgery, School of Dental Sciences, University of Nairobi, P.O. Box 19676 - 00202, Nairobi, Kenya

\title{
TREATMENT INTERRUPTION AMONG HEAD AND NECK CANCER PATIENTS UNDERGOING RADICAL RADIOTHERAPY
}

\author{
M. M. SOLOMON, J. F. ONYANGO, L. O. NYABOLA, A. OPIYO and M. L. CHINDIA
}

\begin{abstract}
Objective: To determine the incidence of treatment interruption among head and neck cancer patients undergoing radical radiotherapy.

Design: Prospective study

Setting: Kenyatta National Hospital (KNH), Nairobi.

Subjects: Twenty $\operatorname{six}(\mathrm{M}=16, \mathrm{~F}=10)$ patients undergoing radiotherapy for head and neck cancer between March and June 2006.

Main outcome measures: Frequency of radiation morbidities and treatment interuptions. Results: There were 26 patients consisting of 16 males and 10 females aged between 21 and 70 years (mean $=49.6$ years). Among these patients $12(46.2 \%)$ had tumours in the oral cavity, six $(23.1 \%)$ had nasopharyngeal tumours, two $(7.7 \%)$ had pharyngeal tumours, and six (23.1\%) had laryngeal tumours. All tumours were primary carcinomas except two pharyngeal tumours which were metastatic. Among the 26 patients, 13(50\%) completed the course of radiotherapy within the prescribed duration while another $13(50 \%)$ had treatment interruption. Of the 13 patients who had treatment interruption, one patient had a treatment gap of four days, seven patients had treatment gaps ranging between six and ten days, and five patients had treatment gaps of over ten days. The duration of treatment gaps ranged between four and 30 days. At the time of treatment interruption the cumulative radiation dose ranged from 22 to 58 Grey with a mean of 38 Grey $($ Mode $=44$ Grey). The most common side effect was xerostomia $(92 \%)$. This was closely followed by mucositis $(88.5 \%)$, skin reactions $(88.5 \%)$ dysphagia $(84.5 \%)$ pain and suffering $(76.9 \%)$. Loss of taste $(61.5 \%)$, trismus $(34.6 \%)$ and voice change (30.89\%) were relatively less common.

Conclusion: Our findings show that the probability of cancer control and cure among head and neck cancer patients treated at KNH could be severely eroded by treatment interruptions as a result of severe radiation morbidity.
\end{abstract}

\section{INTRODUCTION}

The importance of overall treatment time on the efficacy of radiotherapy for cancers, including head and neck cancers, is now well recognised. Over thelast two decades cumulative evidence has demonstrated that prolongation of overall treatment time, whether due to delay or interruption of radiotherapy, is associated with lower survival and locoregional control rates (1- 4).

The biological effects of treatment interruptions (planned or unplanned), on clinical outcome has been attributed toaccelerated tumourstem cell repopulation.
Unlikeother tumourcells, which have limited lifespan, tumour stem cells reproduce indefinitely and can therefore cause recurrence (4). Approximately 2 - 4 weeks after the initiation of radiotherapy, surviving tumour stem cells repopulation in head and neck cancers accelerates and occurs continuously during radiation $(3,4)$. Furthermore, where radiotherapy is interrupted by surgery the accelerated tumour repopulation may be triggered by theenhanced growth environment provided by the healing process (1).

The accelerated repopulation is a tumour response to radiation induced tumour cell death in which the cell cycle of the surviving stem cells is markedly increased 
during thetimes of radiation breaks. During accelerated repopulation the estimated tumour stem cell doubling time may shorten from approximately 60 days to four days (4). The molecular mechanisms underlying accelerated repopulation by tumourcells receiving sublethal doses of radiotherapy likely involves pre-existing signal transduction pathway that have the potential to stimulate cellular proliferation (5).

In light of this evidence, several institutions have reviewed their treatment practices with the view to minimising treatment interruptions or providing dose compensation when the interruptions do occur. Where the interruptions are planned as part of the treatment regime such dose compensation is less complicated. The challenge is in managing unplanned treatment interruptions.

At the moment there is no data on the frequency and causes of interruptions of prescribed course of radiotherapy at $\mathrm{KNH}$. Such knowledge is necessary to determine the need for changes in treatment practice in order to improve clinical outcome. The purpose of this study, therefore, was to determine the frequencies of treatment interruptions and the associated radiation morbidities among head and neck cancer patients undergoing radical radiotherapy at Kenyatta National Hospital.

\section{MATERIALS AND METHODS}

Between March and June 2006 patients undergoing radiotherapy for head and neck cancer wereevaluated for frequency of radiation morbidity and treatment interruptions through interviews, review of clinical notes and selected items of the McMaster University Head and Neck Radiotherapy Questionnaire (HNRQQOL) translated into Kiswahili. The patients were seen every two weeks at the radiotherapy unit and only those patients whose treatments were completed within the study period were considered for analysis. Using a standardised schedule the following demographic and clinical data were collected and analysed; age, gender, site of the tumour, type and incidence of radiation morbidity, number and duration of treatment interruptions and cumulative radiation dose at the time of treatment interruption. The data were summarised by calculating their means, percentages and ranges.

The study was approved by the $\mathrm{KNH}$ and University of Nairobi Ethics, Research and Standards Committee.

\section{RESULTS}

Patient, tumour and treatment profiles: Patient, tumour, treatment and morbidity profiles are shown in Table 1 . There were 26 patients, comprising of 16 males and 10 females with ages ranging from 21 to 70 years ( $M=49.6$ years). Most of the tumours occurred in the oral cavity $(46.2 \%)$ and the rest occurred in the nasopharynx and larynx. All tumours seen were squamous cell carcinoma.

Table 1

Patient, tumour, radiation morbidity and treatment profiles

\begin{tabular}{lcc} 
Parameter & No. & $(\%)$ \\
\hline Age & \multicolumn{2}{c}{$21-70$} \\
Range & \multicolumn{2}{c}{49.6} \\
Mean & \multicolumn{2}{c}{} \\
Gender & 16 & 62.0 \\
$\quad$ Male & 10 & 38.0 \\
Female & & \\
Tumour site & 12 & 46.2 \\
$\quad$ Oral cavity & 6 & 23.1 \\
Nasopharynx & 2 & 7.7 \\
Pharynx & 6 & 23.1 \\
$\quad$ Larynx & 26 & 100 \\
Tumour type & & \\
$\quad$ Carcinoma & & \\
Radiation morbidity & & 92 \\
$\quad$ Xerostomia & & 88.5 \\
$\quad$ Mucositis & & 88.5 \\
$\quad$ Skin reaction & & 76.9 \\
Odynophagia (Pain on swallowing & 84.5 \\
Pain and suffering & & 61.5 \\
Loss of taste & & 34.6 \\
Trismus & & 30.89 \\
Voice change & &
\end{tabular}

Treatment interruptions

$\begin{array}{lll}\text { Yes } & 13 & 50 \\ \text { No } & 13 & 50\end{array}$

Treatment gaps

\begin{tabular}{llc} 
Range & \multicolumn{2}{c}{$4-30$} \\
$<5$ days & 1 & 7.7 \\
$6-10$ days & 7 & 53.8 \\
$>10$ days & 5 & 38.5
\end{tabular}

Of the 26 patients, 13 (50\%) completed treatment within the prescribed duration while another 13 (50\%) had treatment interruptions. Of the 13 patients whose treatments were interrupted, one had a rest period of four days, seven had rest periods ranging between six and ten days and five had over ten rest days. The rest days ranged from four to 30 days. At the time of treatment interruption the cumulative radiation dose ranged from 22 to 58 Grey with a mean of 38 Grey (Mode $=44$ Grey). 
Radiation morbidity: The frequency of radiation morbidity experienced by our patients is shown in Table 1. Xerostomia was the most common side effect followed by mucositis. Voice change was the least common side effect.

\section{DISCUSSION}

The results of this study show a high incidence of treatment interruption however, neither the rate of interruption, nor the frequencies of treatment morbidity are uncommon (6, 7). A study carried out by the Scottish Radiological Society on patients with laryngeal tumour receiving radical radiotherapy found that only $34 \%$ of the patients were treated within the prescribed time while $66 \%$ had their treatment interrupted (7). This rate of treatment interruption is supported by other studies which show that more than $30 \%$ of radical treatments of head and neck cancers are interrupted for various reasons (8-16). Of most concern, however, are the duration and timing of the treatment break. The minimum length of interruption which results in a significant effect on local tumour control is yet to be determined. However, data from split-course therapy studies show that a 14 - 16 day interruption definitely affects treatment outcome (17). Using mathematical models it has been estimated that an unscheduled interruption of one day can result in an absolute reduction of local control of some 1.4\% while an unscheduled interruption of one week can result in a relative loss of local control ranging from $2 \%$ to $25 \%$ (Median $=14 \%)$ for $(18-20)$. This corresponds to a median loss of $26 \%$ in two weeks $(5-42 \%)$. It has been reported that prolongation of a 28-day course by three days or more seriously prejudices both the probability of local tumour control and survival unless the radiation dose is adequately increased (21). Considering these statistics, our findings of treatment interruptions ranging from 4 to 30 days is a major source of concern.

Besides duration, the timing of the treatment interruptions has been reported by some butnotothers as a crucial factor in determining the chances of cure in squamous cell carcinoma $(8,9,22)$. Skladowskiet al. (8) found that gaps at the beginning and the end of the schedule were potentially more harmful than gaps in the middle of the schedule $(8,9,22)$. Harman et al. (9) found that gaps in the second half of the schedule were more harmful than gaps at the beginning of the schedule, supporting the hypothesis that the tumour clonogen repopulation accelerated towards the end of radiotherapy of squamous cell cancer. Robertson et al. (22) on the other hand found no evidence that gaps at the beginning or end of the schedule were potentially more harmful than those in the middle of the schedule. They thus concluded that a gap is a serious adverse event on a radiotherapy schedule irrespective of its position. However, it is not just the length of the unscheduled gap itself which should be cause for concern but also its effect on the overall treatment duration. For example an unscheduled a one-day treatment interruption that falls on the weekend can extend the treatment duration by three days, thus compounding the negative effects of the treatment interruption (22). In this study the timing of treatmentinterruption in relation to weekends and public holidays was not considered. Consequently the actual prolongation of treatment duration could be higher than estimated here.

There are myriad causes of unscheduled treatment interruptions, including radiation morbidity, financial difficulties, difficulties with transport, and machine breakdown. However, the most important of these is radiation morbidity. A treatment regime that results in frequent and/or severe tissue reactions adversely affects compliance as more patients are likely to require prolonged rest days (6). In an earlier study it was found that patients attending radiotherapy for head and neck tumours at the $\mathrm{KNH}$ radiotherapy unit suffered frequent and severe radiation morbidity (23). This may explain, at least in part, the frequent and prolonged duration of treatment interruptions in this study. It is, therefore, prudent to review current radiotherapy regimes at the unit in light of these findings.

In conclusion, our findings show a high frequency and prolonged duration of treatment interruptions among head and neck cancer patients undergoing curative radiotherapy at $\mathrm{KNH}$. However, there is no evidence of compensation for these treatment interruptions. Therefore, the probability of cancer control and cure among head and neck cancer patients treated at $\mathrm{KNH}$ could be severely eroded by the treatment interruptions

We hope that these results will lead to a review of radiotherapy practice at the $\mathrm{KNH}$ with the aim of reducing the frequency and severity of side effects and improving treatment compliance.

\section{ACKNOWLEDGEMENTS}

TotheDirector of MedicalServices, Ministry of Health, who provided the grants for this study, and the Director of Kenyatta National Hospital for allowing us to carry out this study. 


\section{REFERENCES}

1. Russo, G., Haddad, R., Posner, M. and Machtay M. Radiation treatment breaks and ulcerative mucositis in head and neck cancer. The Oncologist. 2008; 13: 1-3.

2. Bese, N.S., Hendry, J. and Jeremic, B. Effects of prolongation of overall treatment time due to unplanned interruptions during radiotherapy of different tumour sites and practical methods for compensation. Int. J. Radiat. Oncol. Biol. Phys 2007; 68: 654 - 661.

3. Taranawski, R., Fowler, J., Skladowski, K., et al. How fast is repopulation of tumour cells during the treatment gap? Int. J. Radiation Oncology. Bio/ Phys. 2002; 54: 229-236.

4. Withers, H.R., Taylor, J.M. and Maciejewski, B. The hazards of accelerated tumour clonogen repopulation during radiotherapy. Acta. Oncol. 1988; 27: 131-146.

5. Schmidt-Ullrich, R. K., Contessa, J. N., Deut, P. et al. Molecular mechanisms of radiation - induced accelerated repopulation. Radiat. Oncol.Investig. 1999; 7: 321-330

6. Vissink, A., Jansma, J., Spijkarvel, F.R.L., Burlage, E.R. and Coppers, R.P. Oral sequelae of head and neck radiotherapy. Crit. Rev. Oral. Biol. Med. 2003; 14:199 - 212.

7. The Royal College of Radiologists. Guidelines for the management of the unscheduled interruptions or prolongation of a radical course of radiotherapy. London. The Royal College of Radiologists. 2002; 1 - 24.

8. Skladowski, K., Law, M.G., Maciejewski, B. and Steel, G.G. Planned and unplanned gaps in radiotherapy: the importance of gaps position and gap duration. Radiother. Oncol. 1994; 30: 109 - 120.

9. Hermann, T., Jabukek, A. and Trott, K.R. The importance of the timing a gap in radiotherapy of squamous cell carcinomas of the head and neck. Strahienther Onkl. 1994; 170: 545 - 549.

10. Duncan, W., MacDougall, H., Kerr, G. and Downing, D. The adverse effects of treatment gaps in the outcome of radiotherapy for laryngeal cancer. Radiother Oncol. 1996; 4: 203 - 207.

11. Harari, P.M. and Fowler, J.F. Idealised versus realised overall treatment times. Int. J. Radiat. Oncol. Biol. Phys. 1991; 29: 209 - 211.

12. Linderberg, R. D., Jones, K., Garner, H.H., et al. Evaluation of unplanned interruptions in radiotherapy treatment schedules. Int. J. Radiat. Oncol. Bio. Phys. 1988; 14: 811 - 815.
13. Bentzen, S.M. Time-dose relationships for human tumours: Estimation from non-randomised studies. In: current topics in clinical radiobiology of tumours. Medical radiology, ed Beck-Bornholt H.P., Berlin: Springer-Verlag 1993 pp 11 - 26.

14. Bentzen, S.M., Johansen, L.V., Overgaard, J. and Thames, H.D. Clinical radiobiology of squamous cell carcinoma of the oropharynx. Int. J. Radiat. Oncol. Biol. Phys. 1991; 20: 1197 - 1206.

15. Bujko, K., Skoczylas, J.Z., Bentzen, S.M., et al. A feasibility study of concomitant boost radiotherapy for patients with cancer of the supraglottic larynx. Acta. Oncol. 1991; 32: 637-640.

16. Report to CRAG on behalf of the Scottish Oncology and Radiology Audit Group (SORAG). An audit of fractionation regimen and treatment gaps in the treatment of carcinoma of the larynx. Edinburgh: Scottish Radiological Society 1997.

17. Overgaard, M., Hjelm-Hansen, M., Vendelbo, J.L. and Anderson, A.P. Comparison of conventional and split-course radiotherapy as a primary treatment in carcinoma of the larynx. Acta. Oncol. 1988; 27: 137 - 143.

18. Barton, N.B., Keane, T. J., Galla, T. and Maki, E. The effect of treatment interruption on tumour control following radical radiotherapy of laryngeal cancer. Radiother. Oncol. 1992; 24: 137 -143.

19. Fowler, J.F. and Lindstrom, M.J. Loss of local control with prolongation in radiotherapy. Int. J. Radiat. Oncol. Bio. Phys. 1992; 23: 457- 467.

20. Lindstrom, M.J. and Fowler, J.F. Re-analysis of the time factor in local control by radiotherapy of T3T4 squamous cell carcinoma of the larynx. Int. J. Radiat. Oncol. Bio. Phys. 1991; 21: 813 - 817.

21. Dale, R.G. and Jones, B. The reduction of tumour control with increasing overall time: mathematical considerations. Br. J. Radiol. 1996; 69: 830 - 838.

22. Robertson, A.G., Robertson, C., Perone, C., et al. Effect of gap length and position on results of treatment of cancer of the larynx in Scotland by radiotherapy: a linear quadratic analysis. Radiother. Oncol.1998; 48: 165 - 178.

23. Solomon, M. M., Onyango, J.F., Nyabola, L. O., et al. Morbidity and quality of life among head and neck cancer patients treated with radical radiotherapy. East Afr. Med. J. 2009; 86: 173-177. 